\title{
Aachen Technology Overview of 3D Textile Materials and Recent Innovation and Applications
}

\author{
Thomas Gries $^{1}$ (D) - Isa Bettermann ${ }^{1}$. Carolin Blaurock ${ }^{1}$. Andreas Bündgens ${ }^{1}$. \\ Gözdem Dittel $^{1}$ - Caroline Emonts $^{1}$ - Valentine Gesché ${ }^{2}$. Nikola Glimpel ${ }^{1}$. \\ Martin Kolloch ${ }^{1} \cdot$ Niels Grigat ${ }^{1} \cdot$ Henning Löcken ${ }^{1}$ - Alexander Löwen ${ }^{2}$. \\ Jens-Lennart Jacobsen ${ }^{1} \cdot$ Magdalena Kimm ${ }^{1} \cdot$ Hannah Kelbel $^{1} \cdot$ Hauke Kröger $^{1}$. \\ Kai-Chieh Kuo ${ }^{1}$. Christoph Peiner ${ }^{1}$. Johannes Sackmann ${ }^{1}$ - Max Schwab ${ }^{1}$
}

Received: 13 May 2021 / Accepted: 7 January 2022 / Published online: 3 March 2022

(C) The Author(s) 2022

\begin{abstract}
This paper provides an overview of the recent definition, technologies and current trends regarding 3D fabrics. In this paper a definition of 3D fabrics, including spacer fabrics, is given and the recent technologies regarding weaving, braiding, weft and warp knitting and tailored fiber placement are presented. Furthermore, an overview of the latest developments in 3D fabrics at the Institut für Textiltechnik of RWTH Aachen University is presented including: large circular 3D knitting, braided and woven structures for medical purposes, newest testing methods and equipment for spacer fabrics, multiaxial fabrics for composites, warp knitted spacer fabrics for space and construction applications, ceramic matrix composite 3D braiding and 4D textiles.
\end{abstract}

Keywords 3D fabrics $\cdot 3 \mathrm{D}$ textiles $\cdot 4 \mathrm{D}$ fabrics $\cdot$ Spacer fabrics $\cdot$ Technology overview Current research $\cdot$ Woven $\cdot$ Knitted $\cdot$ Braided

\section{Introduction-Advantages and Challenges of 3D Textile Technologies}

3D textiles have undergone rapid development in recent years and have extended the scope of applications for textile materials to products in automotive, aerospace, and other such industries. Significant technical developments such as introduction of digital design based approaches, automation and near net shape manufacturing have been made across all steps of the process chain from preliminary design to application.

For more than 30 years, Institut für Textiltechnik of RWTH Aachen University (ITA) has been researching and developing 3D textiles. Machines and technologies as well as new products and simulation models have been and are being investigated and further developed in Aachen.

Thomas Gries

Thomas.Gries@ita.rwth-aachen.de

Institut Für Textiltechnik, RWTH Aachen University, 52074 Aachen, Germany

2 PerAGraft GmbH, 52070 Aachen, Germany 
The usage of 3D textiles offers considerable advantages for many applications: less material consumption, continuous and consistent yarn architecture and less production steps. In contrast to this, the productivity compared to regular $2 \mathrm{D}$ fabric production is reduced by 5 to 10 times while machine investment and process costs are increased by 5 to 10 times due to complex machine parts and increasingly demanding machine handling. Currently, there are no CAD-Models or sufficient digital tools that enable the simulation of the mechanical properties of 3D fabrics. Therefore, the product and application development process for 3D fabrics is costly and slow. In addition to that, there is a lack of standardized testing methods for 3D fabrics which leads to a lack of comparison between 3D fabrics and 2D fabrics or non-textile materials.

In the following, a definition of 3D fabrics will be given, the main production techniques of 3D fabrics will be presented and some highlights of the current research at ITA will be shown.

The difference between regular 2D fabrics and 3D fabrics can be defined in different ways and rarely is there a strict boundary between 2 and 3D fabrics. The reason for this blurred boundary is that all physical objects are formed in 3 dimensions. At most, it happens that at 2D textile has no designed expression of a yarn or fiber in the $\mathrm{z}$-axis, hence the third dimension.

Fabrics called 3D fabrics have characteristic values in all 3 physical dimensions. The 3D shape of these fabrics can be created by yarn architecture or textile architecture. Yarn architecture is used to describe the arrangement of yarns inside a fabric. The fabric is defined as a 3D fabric based on its yarn architecture if it is formed by three or more yarn systems and no rectangular coordinate system can fit into the fabric while maintaining a perpendicular position to each yarn system. For example, spacer fabrics are typically considered 3D fabrics due to their yarn architecture.

The textile architecture describes the geometry of the fabric. A fabric is defined as a 3D fabric in terms of textile architecture if a volume is formed or created by the fabric; therefore, socks are typically regarded as 3D-fabrics due to their textile architecture $[1,2]$.

\section{Technologies for the 3D Fabric Production}

\subsection{D Weaving Utilizing Textile Architecture}

As stated in the introduction, 3D woven fabrics can be defined by two main categories: yarn vs fabric architecture. These categories can in turn be further broken down into further sub-categories including 3D solid, 3D hollow, 3D shell and 3D nodal and these can in turn be broken down and so on and so forth [3]; however, due to the fact that the different technologies can be utilized in combination with one another, a clear distinction between these categories is not always possible. For example, currently ITA is developing a variety of 3D woven preforms utilizing both fabric and yarn 3D architecture. In the example found in image Fig. 1, the walls of the structure consist of a multiple layer woven structure, which

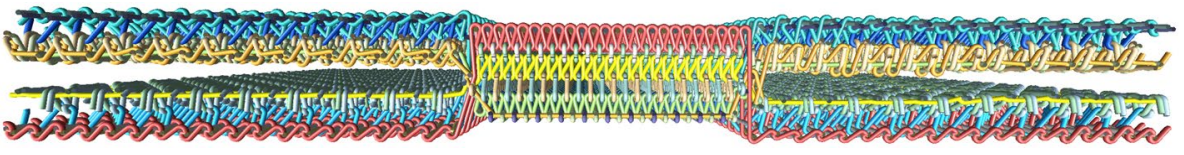

Fig. 1 3D Rendering of a carbon fiber composite preform concept [4] 

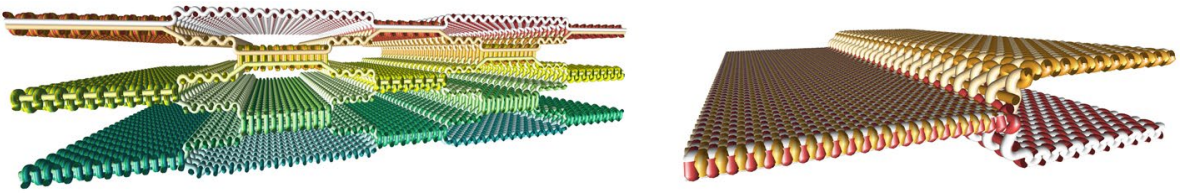

Fig. 2 3D woven composite parts: honeycomb multilayer fabric (left), T profile (right) [4]

is used in combination with a hollow structure to produce the necessary structural openings and dimensions for the final preform structure.

Woven fabric with a 3D textile architecture such as 3D hollow in which tubes and pockets are formed within the structure and 3D nodal in which tubes are formed and connected [3], are particularly interesting for the composites sector due to their special mechanical properties. When utilizing 3D weaving technology, multiple 2D surfaces are manipulated in such a way that the various layers of fabric are connected during the weaving process, producing a flat cloth that can be unfold to reveal a 3D structure. This process produces a 3D woven structure that requires little to no subsequent modifications, such as cutting or joining. This complex textile architecture results in a direct force transmission throughout the connection of the surfaces. In addition, the lack of cutting reduces the amount of material used.

Although multiple layer and even 3D fabrics can be woven on traditional weaving machines, special weaving machines for fabrics of high thickness have been developed which further expands the range of applications and reduces the number of required finishing steps. The shuttle weaving technique is often used with several shuttles at the same time. Utilizing a shuttle produces a fabric with closed edges which further reduces the need for subsequent finishing processes and produces a more complete 3D structure. Rapier insertion can also be used to produce 3D fabrics. This technology is much faster than shuttle weaving, but the weft threads are cut at the edges which can be a disadvantage, depending on the application.

\subsection{D Weaving Utilizing Yarn Architecture}

3D solid fabrics are a weaving standard and are traditionally used in the creation of everyday commodity items such as blankets and upholstery fabrics. As technology has progressed, multiple layer fabric have become common place in various technical fields such
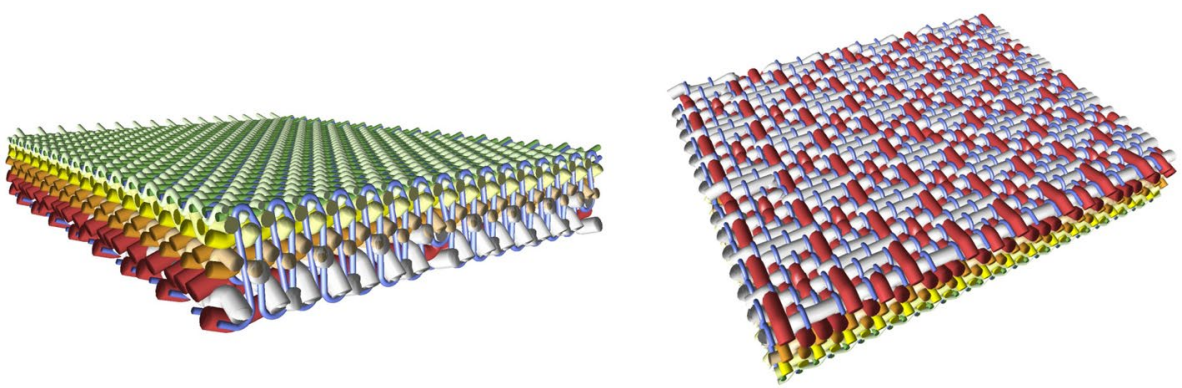

Fig. 3 3D rendering of a multiple layer structure with varying surface structures show the face (left) and the back (right) of the woven structure [4] 

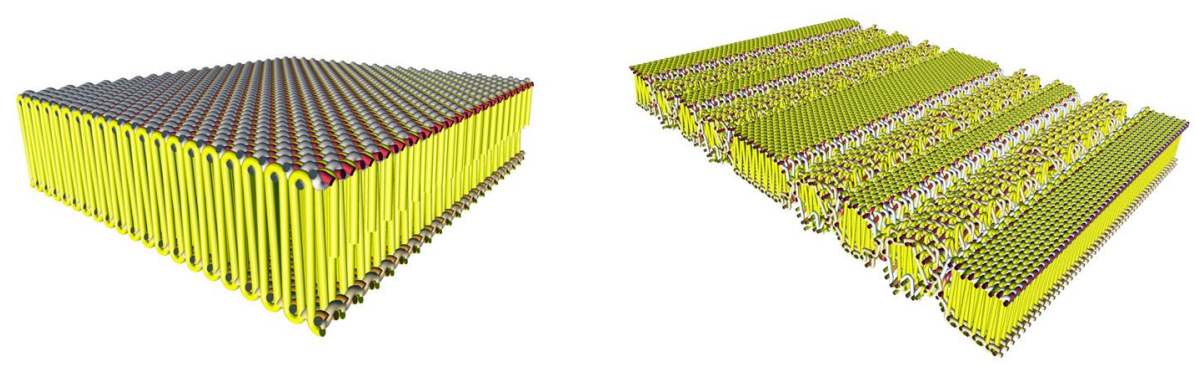

Fig. 4 3D renderings of different spacer fabrics [4]

as, protection, composites and medical to name a few. For example, in the production of paper a conveyor belt is needed that is smooth and even on the top side, but open-pored on the bottom side which is achieved using a multiple layer woven structure.

3D solid fabrics are formed by allocating the warp ends to specific layers and then inserting weft picks in a specific sequence that allows for different layers to be formed. Additionally, a binding system must be defined or integrated to create a connection between the layers and to form the final solid fabric. This process is extremely versatile due to the fact that each individual layer and binding methods can be separately designed to tailor the technical properties to the desired end use. Using a multilayer structure also allows for a much higher warp and weft density as compared to a single layer fabrics. In Figs. 2, 3, 4 , and 5, the rendered fabric is multi-layered with an orthogonal binding and independent woven structures on the face and back of the fabric to create a smooth structure on the face and a textured surface on the back of the fabric.

Woven spacer fabrics are specialized type of multi-layered fabric in which two outer layers of fabric are formed using two independent warp systems with a third warp system that moves through the outside layers to form a 3D spaced fabric. They have a variety of applications including filtration, cushioning, inflatables and heat wicking.

For the production of 3D spacer fabric, special weaving machines are required in which the warp threads, from which the spacer threads are formed, are pulled off the warp beam during the weaving process independently of the threads forming the faces and two weft systems are inserted simultaneously to retain the required height of the spacer threads. Standard weaving machines with an additional warp beam can be used for spacer

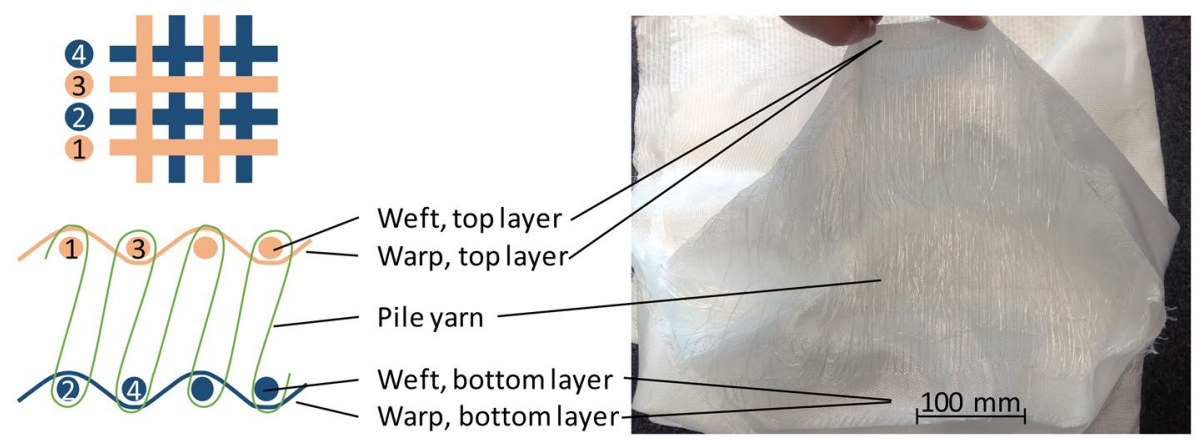

Fig. 5 Schematic drawing of a woven spacer fabric with pile yarn (left) and sample of a woven spacer fabric with long distance pile yarn (right) [4] 
fabrics with a small distance between the top and bottom layers but quite often the distance between layer can be significant. For inflatables (e.g. inflatable boats and water tanks) the distance between layers can be well over $20 \mathrm{~cm}$.

\subsection{Woven Tubular Structures}

Woven tubular structures can be produced by weaving a double layer flat weave whose layers are interconnected at the edges through an appropriate weaving pattern. By pulling the tubular weave over a mandrel with the desired cross sectional shape the weave obtains that shape. All kinds of shapes (e.g. triangular, rectangular, polygonal etc.) are conceivable as long as the perimeter of the weave complies with the perimeter of the shape (Fig. 6). For maintaining the shape, the mandrel either remains inside the woven tubular structure or the setup is heat-set and the mandrel is removed afterwards. The most common woven tubular structure is the circular tube.

Woven tubular structures can be produced either on a narrow needle loom or a narrow shuttle loom (see Fig. 7). Despite the significantly lower productivity of the shuttle weaving technique (approx. 200 wefts/min) compared to the needle weaving technique (approx. 2000 wefts/min.) the shuttle loom is still preferably used due to its possibility for weaving 'real' or 'closed' selvedges. These allow the shuttle woven tubes to have a continuous, seamless, closed structure throughout the whole circumference. Woven tubes produced on needle looms have a knitted selvedge on one edge of the weave. Thus, they show a locally higher porosity on the edges which are weak points for leakage and mechanical failures [5].

Another way of weaving continuous, seamless, tubular structures is the 3D circular weaving method where the warp yarns are arranged circumferentially and inserted along the axial direction. The weft yarn is then inserted by a shuttle running in circumferential direction through the shed which is formed by the warp yarns in the shape of a helix. This method has high production speeds due to the steady motion of the shuttle. However, small tube diameters are not feasible due to the size of the shuttle [6].

Woven tubular structures are used in construction composites (e.g. fiber reinforced tubes for tubular structural components), chemical industries (e.g. tubular filters) and in medical textiles (e.g. artificial vessel prostheses).

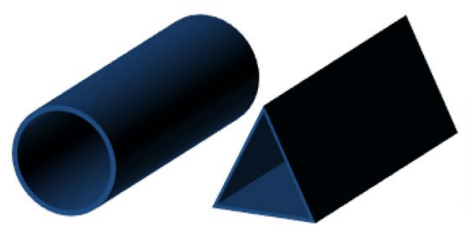

a) b)

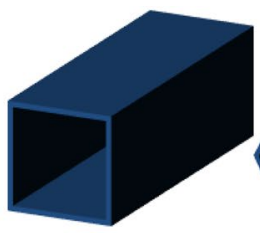

c)

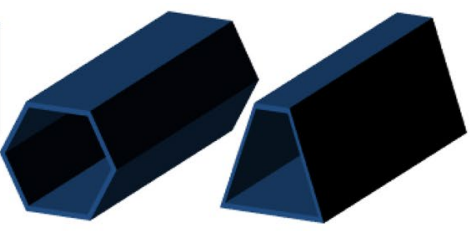

d) e)

Fig. 6 Examples of woven tubular structures; a) circular, b) triangular, c) rectangular, d) polygon, e) trapezoid [4] 

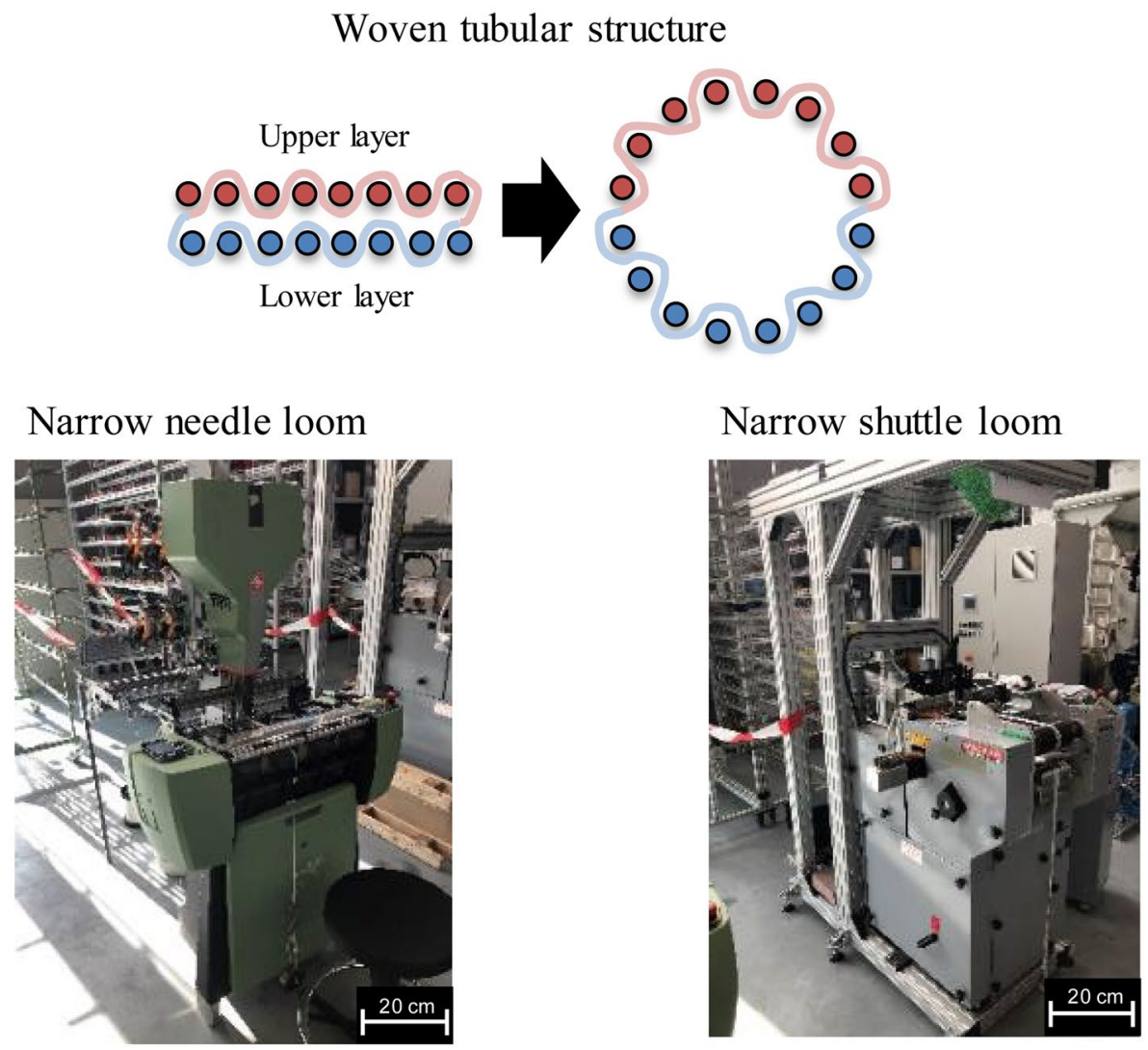

Fig. 7 Woven tubular structure and schematic design of a needle loom (left) and a shuttle loom (right) [4]

\subsection{D Flat Knitting}

3D knitted textiles can be produced in various shapes on flat bed knitting machines. Due to the high flexibility of the flat knitting machines, the production of spacer fabrics, near-end shape textiles, tubes and other forms of 3D textiles are possible to knit. With the production of near-net shaped fabrics, a majority of the subsequent manufacturing processes can be eliminated and a zero-waste approach in the production can be realized [5].

The shaping of textiles on flat knitting machines is achieved by varying the number of stitches in the wale and/or course direction and by varying the stitch length. For the variation of stitches, the following techniques are used: widening, narrowing, bind-off and partial knitting. For the widening of a knitted fabric additional needles at the edges are activated. For narrowing, the needles on the edges are deactivated. During the activation/ deactivation of needles the loops from the outer needles are constantly transferred to the adjacent needles. By transferring the loops, the edge of the fabric is tailored to the desired width. The bin-off is a special type of narrowing to create a fixed edge at the end of a knitted fabric. With widening, narrowing and bin-off it is possible to create a near-net shape textile $[5,7]$. 
For the creating of a 3D shape in flat knitted textiles, partial knitting and the variation of the stitch lengths are used. To create a 3D shape with partial knitting, some needles with their active loops are parked during the knitting process. With this needle parking, the knitted fabric can be shaped. The process is similar to integrating darts during traditional clothing manufacturing and sewing. To further support the creation of the desired 3D shape, the stitch length in some areas can be adapted. Due to their high flexibility, a wide range of 3D shapes can be produced on flat knitting machines. Because of the rather complex programming, these 3D forms are still mainly used in the clothing industry for socks, gloves, sweatshirts or shoe upper. Nevertheless, an increasing number of technical textiles are also being produced with a 3D shape on flat knitting machines.

\subsection{Weft Knitted Spacer Fabrics (Circular and Flat)}

Weft knitted spacer fabrics can be produced on flat bed knitting machines and on double bed circular knitting machines. The weft knitted spacer fabrics are consisting of two knitted layers and are connected with a pile yarn (see Fig. 8). For the production of weft knitted spacer fabrics, three independent yarn systems are needed, one for each outer layer and the pile yarn. The knitted layers are each produced on one knitting bed, independently from each other. The pile yarn is inserted alternating in both layers using tuck stiches. Due to the higher stiffness of the pile yarn the layers are held at a distance in the produced fabric [5, 8].

The main advantages of weft knitted spacer textiles are the rather short machine set up, economic production and their properties regarding air permeability and a soft touch. The distance between the two layers in weft knitted fabrics is limited to a few millimeters. Due to the independent knitting of the top and bottom layer the pattern on both knitted layers can be designed independently. Weft knitted spacer fabrics are used for a variety of applications, such as mattress covers, car headliners or clothing [5,8].

\subsection{Warp Knitted Spacer Fabrics}

Warp knitted spacer fabrics are produced on double needle bar Raschel machines (see Fig. 9). The fabric, that can either be a single fabric, two fabrics, a tubular structure or a spacer fabric is drawn downwards in the needle gap [9].

Fig. 8 Weft knitted spacer fabrics [4]

\section{Upper surface}

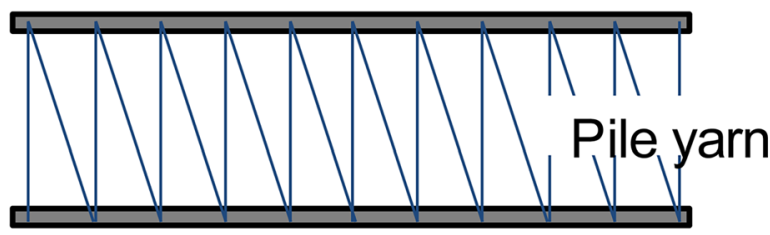




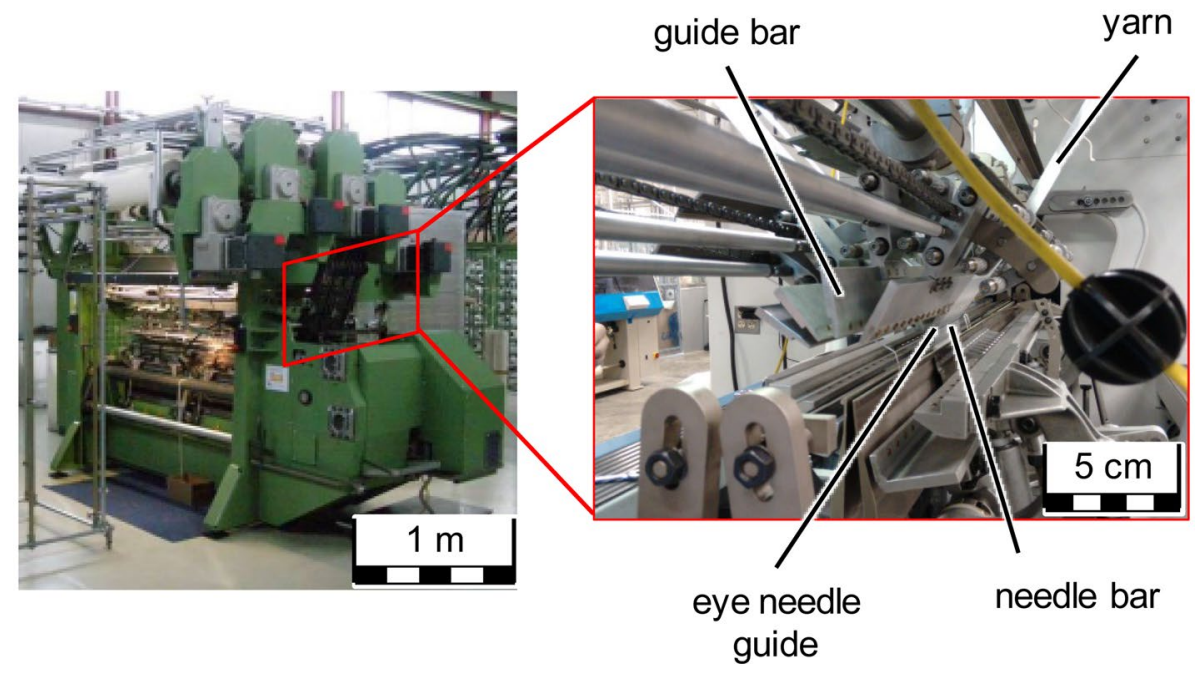

Fig. 9 Design of a double needle bar Raschel machine [4]

Spacer fabrics consist of two parallel knitted fabrics, which are connected to each other in the z-direction by so-called pile yarns. The basic structure of the cover surfaces consists of knitted fabrics, so that a fundamentally high elongation and thus drapeability is achieved. The pile yarns integrated in the z-direction give the overall structure the necessary stiffness. As a half-finished textile product, spacer fabrics can be (temporarily) deformed or folded without destroying the structure [5].

Applications for spacer textiles can be found wherever increased air circulation or a comfort effect due to the cushioning structure is desired [10]. They are used in medical textiles (e.g. orthotics), in automobiles (e.g. climate comfort layer), in home textiles (e.g. chair cover), in clothing (e.g. bras) as well as in protective and sports clothing [11].

There are different types of 3D warp knitting machines, with which we can produce (dendritic) tubular structures and spacer fabrics. For example, 3-dimensional cell carriers for tissue engineering, small-lumen vascular prostheses or aortic prostheses with outlets for the leg arteries can be warp knitted.

\subsection{D Braiding}

3D braiding technologies enable the production of structures with complex geometry, which are often used for lightweight solutions, for example in automotive engineering. In addition, medical technology offers wide-ranging applications for 3D braiding technology. 3D braided structures are defined as those with yarns that intersect in all three spatial directions. 3D braiding processes allow the fiber orientation to be easily influenced, thus ensuring high strength and stiffness with reduced mass [5, 12].

In the process of rotary braiding, the bobbins are moved on two concentric circular paths with opposite directions of rotation. To achieve the crossing of the yarns, both circular paths are superimposed by $180^{\circ}$ phase-shifted sinusoidal oscillations which intersect regularly in crossing points every $180^{\circ}$. The bobbins alternate at each of these crossing points between the outer and inner slide ways during a cycle and thus generate the under- and overlapping of the yarns. This basic principle of rotary braiding can be used to over-braid 
a core, on which the yarns are placed. Thus, as more layers of the braided preform are produced, a tubular reinforced 3D-product is generated.

The fully digitized 3D braiding machine at ITA with Industry 4.0 standard enables the near-net-shape production of three-dimensionally braided textile preforms. Depending on the application, the preforms products produced with this technology can be specifically reinforced in all three spatial directions and can also be modified in cross-section during the process. The resulting high degree of design freedom for preforms enables complex 3D-structures to be produced in just one process step.

At ITA, 3D hexagonal braiding technology is used in the field of medical textiles. The machine was developed in cooperation with the University of British Columbia and technically improved in several iterations. A special aspect of the hexagonal braiding technology is the tight packing of the machine bed. The lace braiding mechanism allows two yarn carriers to take up the position between two horn gears, maximizing the number of carriers. Due to the reduction of distance to the braiding point, the process is especially suitable for fine materials. One of the major applications is the near-net shape production of tubular structures and ramifications (Fig. 10).

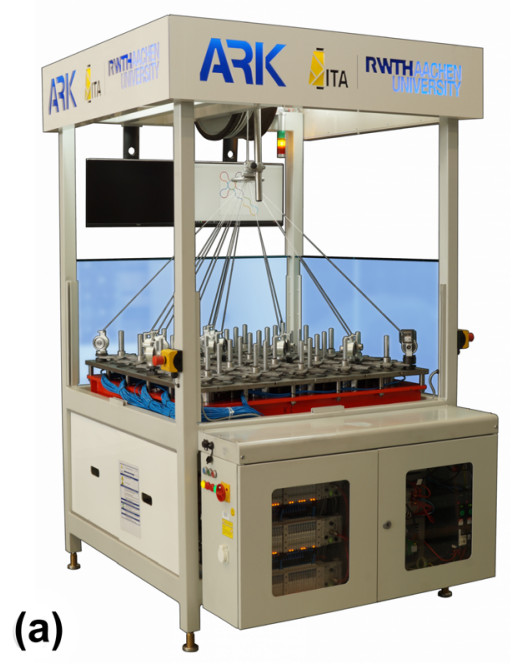

(c)
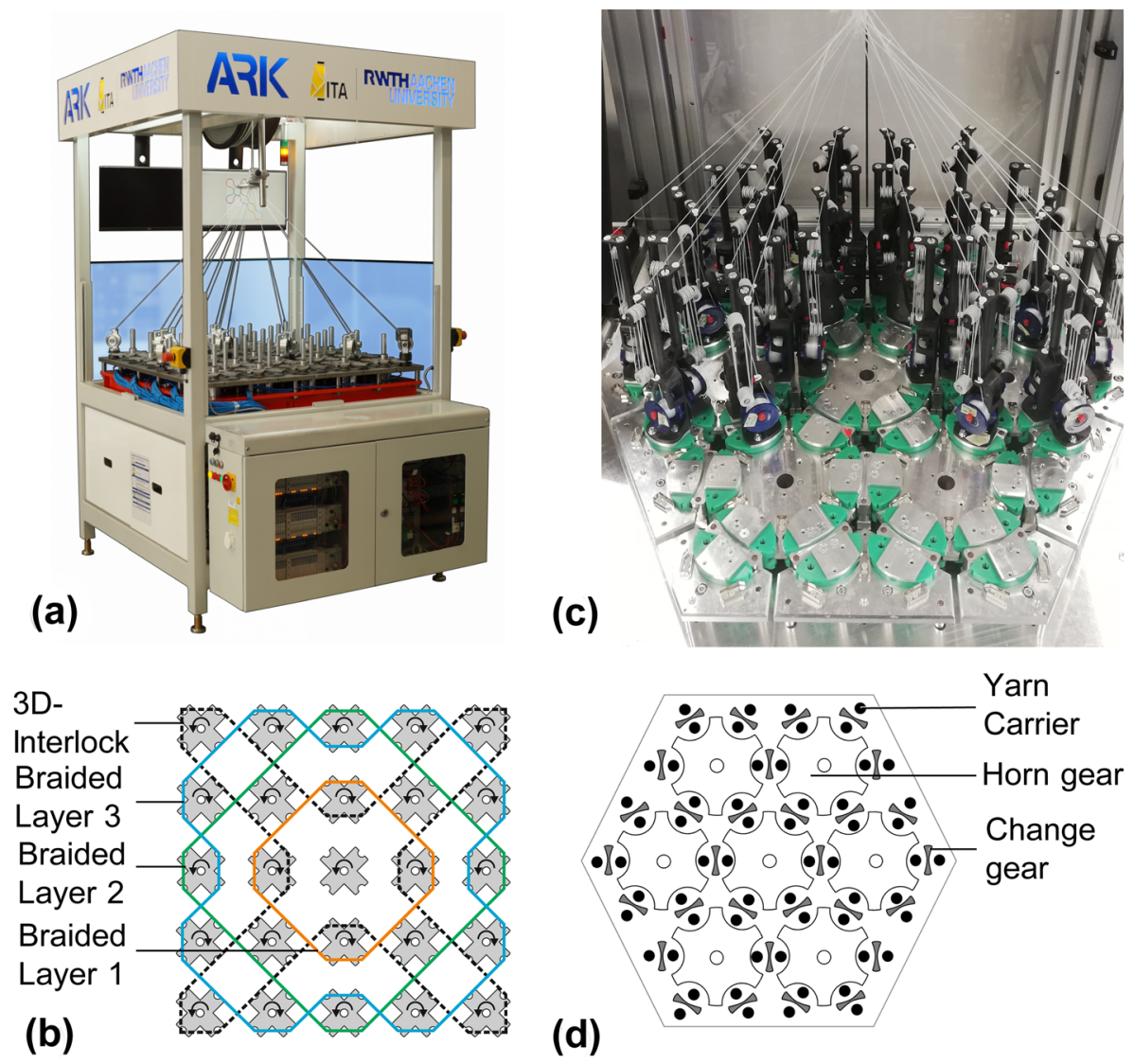

Fig. 10 3D Braiding Technology at Institut für Textiltechnik of RWTH Aachen University. (a) fully digitized 3D braiding machine, (b) machine bed with exemplary multilayer 3D braiding pattern, (c) 3D Hexagonal Braiding machine, (d) hexagonal shaped machine bed [4] 

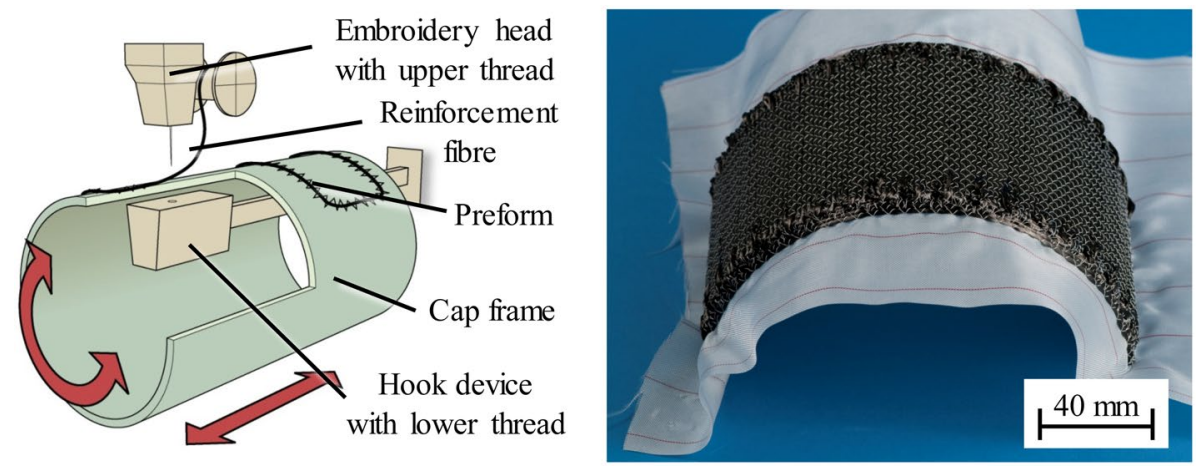

Fig. 11 left: Principle of 3D Tailored Fiber Placement [13], right: produced preform on the machine modified at ITA [14]

\subsection{D Tailored Fiber Placement (TFP)}

The TFP technology is a technical embroidery technology which allows fiber placement along main load paths and near-net shaped preforms for fiber reinforced composites. The principle is derived from embroidery, where upper and lower thread are generating doublelock stitches which then fixate the reinforcement fibers onto the substrate. The entire process is two dimensional (see Figs. 6, 10, 11). Therefore, subsequent draping or forming of the preform can lead to artefacts, such as folds or undulations [13].

At the ITA the 3D-TFP has been developed within the IGF funded Project ("Basic textile research on the design of three-dimensional tailored composite parts" No. $15475 \mathrm{~N}$ ) from 2008 to 2010 . The embroidery machine has been modified by installing a cap frame (see Figs. 7, 12). This module has been customized so fibers can be placed on a cylindrical substrate. The substrate itself can then rotate from left to right and move front to back [14]. The components can also be produced three dimensionally in just one step. Results show that the direct 3D embroidery generates less defects within the textile and no subsequent draping is necessary [13]. Although the technology is currently only feasible for cylindrical shaped parts, new concepts were developed within the project to manufacture truly 3D shaped preforms [13]. One of the biggest challenges within this
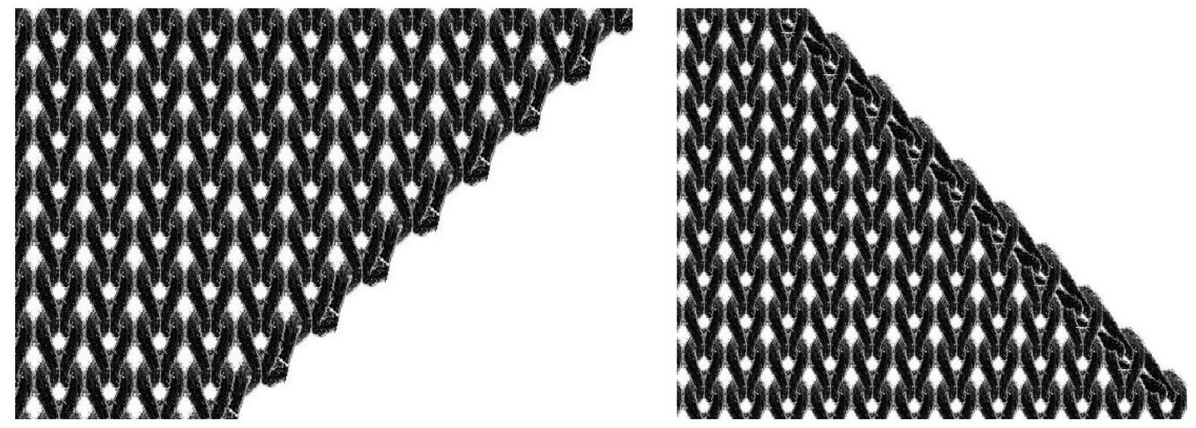

Fig. 12 Shaping of 3D fabrics through widening and narrowing [4] 
technology is the substrate which has which simultaneously has to be rigid enough for a $3 \mathrm{D}$ fiber placement but can also be penetrated by the needle.

\section{Current Developments on 3D Textiles at ITA}

\subsection{D Large Circular Knitting}

The ITA 3D-Knit Technology was developed by Dr. Kristina Fuhrmann at the Institut für Textiltechnik of RWTH Aachen University (ITA). The basic principle was presented at the 8th World Conference on 3D Fabrics and their Applications in Manchester, UK on the 28-29th of March 2017. The ITA 3D-Knit principle enables the production of 3D shaped knitted fabrics while still using the high productivity of the continuous needle movement of large circular knitting machines. There are no machine modifications needed and the productivity of large circular knitting machines can be enhanced with the new technology. As it is generally the case with 3D knitting, the prerequisite for this application is individual needle control, which means that a jacquard machine is required [7, 15-18].

To enable the production of 3D-knitted fabrics on large circular knitting machines, a new knitting pattern was developed. With this pattern, the implementation of a reduction of the surface, which is typically made by confection during the cut \& sew process, is enabled. This "sewing-like" knitting pattern (or darts) can be integrated into the knitted fabric to achieve the desired three-dimensional form. The knitting pattern consists of floats and stitches that alternate horizontally over the area to be reduced. Due to the continuous stitch wales of floats, the corresponding needles are not moved in this area and thus hold the knitted fabric in position. The other needles continue to form loops, but produce these on the backside of the knitted fabric. In this way, the continuous movement of the circular knitting machines can be maintained and the effective surface area can be reduced [15-18].

Although the ITA 3D-Knit Technology enables the production of 3D knitted fabrics, it is not possible to achieve the same high flexibility of flat knitting machines. It is still necessary to cut the fabric out of the knitted tube and the reduced fabric remains on the backside, and therefore does not reduce the amount of material used [15-18].

\subsection{D Braiding in Medical Applications}

Current applications of the 3D hexagonal braiding technique include synthetic ligaments as well as complex stent structures. Synthetic ligaments show deficits regarding mechanical long-term performance. Therefore, the current gold standard are autologous grafts. These have limited availability and increase the risk of donor-site morbidity. By using degradable polymeric yarns tissue engineering scaffolds, tendon regeneration and augmentation can be achieved. 3D braids offer improved mechanical properties i.e. maximal tensile force and stiffness, due to the interconnection of the different braid layers. The dense packing of the machine bed enables a particularly gentle fiber processing of degradable and fine 
Fig. 13 Examples of 3D Hexagonal Braiding; (a) bifurcated Nitinol stent, (b) synthetic ligament [4]

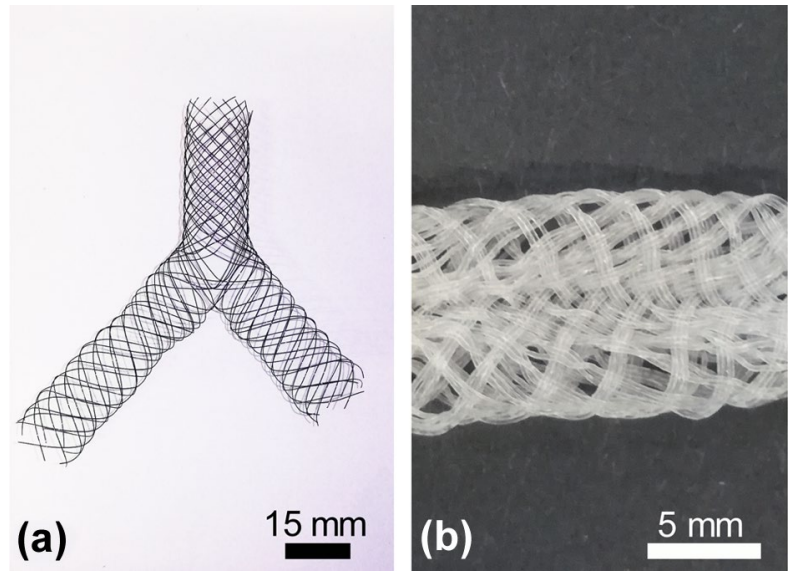

polymer materials. The fibers can serve as a guide structure for cells due to three-dimensional deposition.

Tumors in bifurcations in e.g. the respiratory tract have to be treated extensively, for example by double stenting. Self-expanding bifurcation stents made of Nitinol allow a simplified treatment. By 3D hexagonal braiding these stents can be manufactured in one process step. The braiding angle, number of filaments and bifurcation angle can be adjusted individually. By switching between different braid geometries e.g. round braid and flat braid, holes can be introduced into the structure to accommodate the patient's individual anatomy. Further applications can be occlusion devices and blood filters for the venous system (Fig. 13).

\subsection{D Warp Knitting in Medical Applications}

Warp knitting technology is particularly suitable for the production of 3-dimensional structures for use in medicine. Mechanical and geometrical properties can be precisely adjusted via material selection, combination of different materials or yarn counts and by different process parameters.

The current research at the Institut für Textiltechnik of RWTH Aachen University aims at avoiding compliance mismatches between the artery and the graft (Fig. 14). A compliance mismatch is a difference in the stress-strain behavior of the artery and the graft. Especially in small caliber vascular grafts the compliance mismatch leads to insufficient patency rates. The current research aims for the development of a textile-based vascular graft with physiological compliance properties. To achieve this goal, a biomimetic approach is chosen: by combining elastic and non-elastic fibers in a tubular warp-knitted structure, a combination of material elasticity and structural elasticity in grafts is aspired.

Furthermore, warp knitting can be used as a platform technology for the production of textile reinforcement structures for hydrogels in tissue engineering applications. Due to their high-water content and their cell adhesion motifs, hydrogels offer a good biological environment for cell growth. Additionally, the structure of hydrogels resembles the structure of the extracellular matrix in the human body. However, hydrogels show poor mechanical properties. Warp knitted spacer textiles can be used to compensate for these 
Native vessel:

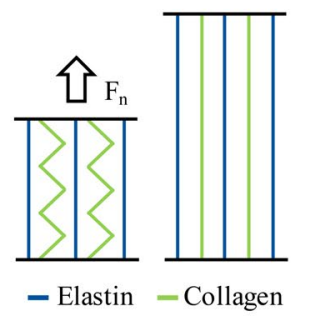

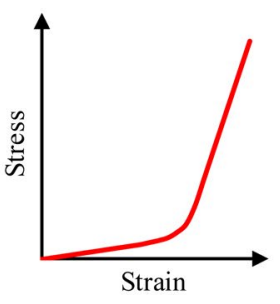

Graft:
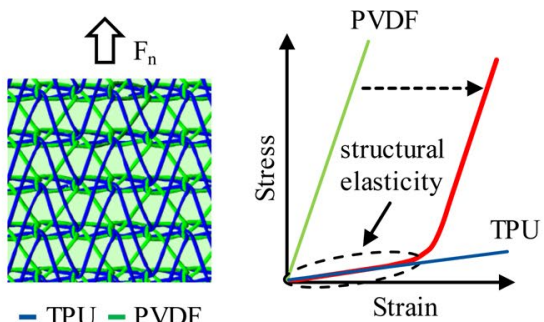

Strain

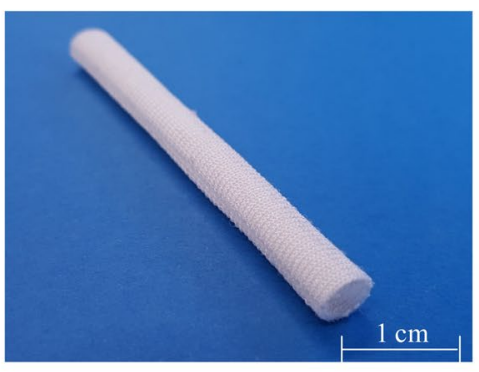

Fig. 14 Synthetic vascular graft with reduced compliance mismatch [4]

disadvantages. The pore size and porosity as well as the mechanical properties of warp knitted spacer fabrics are well adjustable. In contrast to the hydrogels, however, they do not create a favorable biological environment for the cells. By filling the spacer fabrics with a hydrogel, the advantages of both materials can be combined resulting in a hybrid scaffold with favorable biological properties, as well as adjustable mechanical properties. By adjusting the mechanical properties of the hybrid scaffold, a variety of tissue engineering applications for different human body tissues can be addressed.

\subsection{Patient Individualized Implants Using 3D Fabrics}

Clinically established implants are usually off the shelf products available in certain standard sizes. Medical-technological progress, especially in the field of medical imaging and emerging production technologies as e.g. textile technologies have recently enabled the realization of custom-made solutions. The challenge is to translate the medical image data from CT or MRI scans into the patient specific implant design. This design may include geometrical as well as functional individualization parameters. This process requires the construction and development of robust and highly flexible parameter-based CAD models. In a second step, these CAD models need to be translated into a textile machine code, to enable a fast and effective production of the implants. The decisive factor in choosing a suitable textile technology is the digital interface on the machine side. Textile technologies which allow for a high adaptability and variability in the producible structures are predesignated for such a task. Examples are narrow weaving, flat bed weft knitting and warp knitting, as these technologies are capable of producing tubular fabrics and three-dimensional 
fabrics. It is the holistic approach to combine the prediction of textile behavior and the extraction of geometrical design parameters from the medical image data into robust models that leads to the vision of cost-efficient and fast delivery of patient individualized implants.

This is the aim of PerAGraft, a spin-off of the Institut für Textiltechnik of RWTH Aachen University. The team develops patient-individualized textile implants for cardiovascular applications. The implementation of an end-to-end digital process chain is at the core of its innovation to produce textile-based patient specific implants.

\subsection{Tubular Weaving in Medical Applications}

The most popular 3D woven textile for medical applications is the tubular woven conduit. Woven conduits are preferably used for vascular implants. Due to their adjustable pore size and porosity, which can achieve very small values, they can be woven as water- and blood-tight structures without additional coatings. Furthermore, woven conduits show an excellent high tensile strength behavior in all directions which gives the implants a durable shelf life. However, they lack elastic behavior to give a necessary flexibility to the implants. Also, woven conduits have a low kink-resistance due to their comparatively stiff behavior. A way to counteract the disadvantages is to pleat the weaves. This accordion-like shaping of the fabric gives the woven conduit the necessary elastic and kink-resistant characteristic for the use as vascular grafts. In case of stent grafts, metal stent structures are sewn on the woven conduits to keep the hollow lumen open while bending.

At the Institut für Textiltechnik of RWTH Aachen University, a currently ongoing research project broaches the issue of ultra-low-profile woven stent grafts for treating abdominal aortic aneurysms in patients with strongly angulated and narrowed access vessels. The aim of the project is to reduce the folded profile of the woven stent graft by using thin-walled woven conduits. Hence, a better access through the complicated femoral vessels can be enabled. For this purpose, ultra-fine medical-grade multifilament yarns $(\leq 20$ dtex) are used for weaving the thin-walled woven conduit. To guarantee the impermeability against blood leakage, an extremely tight woven structure with high yarn densities is required. This requirement leads to several difficulties such as yarn clamping during the shedding and yarn breakage in the weaving process which this project is going to address.

\subsection{Testing Equipment and Methods for Spacer Fabrics}

Spacer fabrics are three-dimensional textile structures consisting of two textile cover surfaces and a spacer thread. The cover surfaces are kept apart at a distance by the spacer thread. This structure gives spacer fabrics special properties that make them a suitable substitute for other, non-textile materials. Applications can be found wherever increased air circulation, spacing between cover surfaces or a comfort effect due to the cushioning structure is desired. Due to their special properties, spacer fabrics differ from conventional, flat textiles. Up to now, spacer fabrics have been tested according to standards for flat textiles or other non-textile materials. However, these standards do not take into account the special requirements for testing spacer fabrics. Therefore, objective comparisons among spacer fabrics or between spacer fabrics and conventional flat textiles or non-textile materials cannot be made in all areas of application. In a publicly 
funded joint project, the Institut für Textiltechnik of RWTH University (ITA) has developed testing devices and methods in cooperation with industrial partners and has transposed them into standardization documents in cooperation with the German Institute for Standardization (DIN e.V.). The material properties taken into account are for maximum force, thickness, compression, mass per unit area, definition of terms and sample preparation. Within the new testing standards, the special properties of spacer fabrics are given special consideration. This enables standardized testing of these properties and thus objective comparisons not only between spacer fabrics themselves but also, with conventional flat textiles and with non-textile materials.

In order to enable standardized testing of spacer fabrics and thus objective comparisons of spacer fabrics with each other, with conventional flat textiles and non-textile materials, further research is needed. It will be necessary to develop and validate test equipment and procedures for the determination of further properties of spacer fabrics, e.g. compression hardness, compression set, tilt stability, pressure point distribution, abrasion test and maximum tensile strength. Furthermore, since these results are mostly developed to fit the requirements of warp knitted spacer fabrics, the research results should be evaluated regarding weft knitted and woven spacer textiles.

\subsection{Multiaxial Fabrics for Higher Fatigue Strength of Composites}

Glass fibre-reinforced plastics (GFRP) are an ideal material for leaf springs due to their very high energy absorption capacity. GFRP leaf springs are used in both machinery and automobiles [19]. The use of GFRP as a spring material imposes a dynamic load on the material, making fatigue strength a key design criterion [20]. A GFRP leaf spring consists of individual glass fibre layers. Most of the layers are oriented in the longitudinal direction of the spring $\left(0^{\circ}\right)$ and serve as load-bearing elements. However, for manufacturing reasons, also layers in the transverse direction of the spring $\left(90^{\circ}\right)$ are present in the laminate. The transition between the $0^{\circ}$ and $90^{\circ}$ glass fibre layers is one of the places in the laminate where fatigue fractures occur. The fatigue fractures appear as delamination between the $0^{\circ}$ and $90^{\circ}$ layers, which in turn can occur because the $0^{\circ}$ and $90^{\circ}$ layers are only connected in the thickness direction by the relatively weak polymer matrix.

Within the framework of ongoing research projects at ITA (Fig. 15), it is being investigated how fatigue fractures at the layer transition can be prevented using hybrid multiaxial fabrics. For this purpose, the previous individual layers in the $0^{\circ}$ and $90^{\circ}$ directions are combined in a multiaxial orthogonal fabric. Within one fabric layer, three fibre directions are combined: $0^{\circ}, 90^{\circ}$ and $0^{\circ}$. The orthogonal fabrics are manufactured in

Laminate with multiaxial-farbric layers

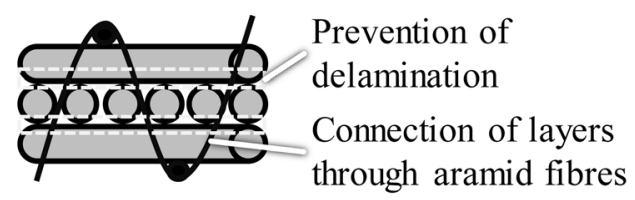

Laminate with separate layers

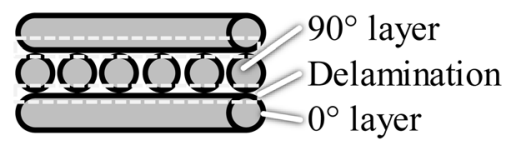

Fig. 15 Prevention of delamination through multiaxial fabrics 
such a way that the glass fibres are almost free of undulation. They are connected by an additional thread system composed of aramid fibres. The use of a multiaxial fabrics enables a connection of $0^{\circ}$ and $90^{\circ}$ layers with load-bearing fibres in thickness direction, thus counteracting the occurrence of delamination.

However, fatigue fractures are also a result of the accumulation of previous micro damages. One of the critical damage mechanisms are fibre breaks. Therefore, additional investigations are being carried out to determine how the formation of fibre-break clusters can be prevented. The approach is to hybridise individual laminate layers: Glass fibre rovings are partially replaced by crack arresting fibres. The crack arresting fibres are characterized by a high elongation at break or a high fracture toughness, which prevents the formation of fibre fracture clusters. Polyamide-, UHMWPE-, Aramid- and Polyesterfibres are investigated for this purpose.

\subsection{D Warp Knitted Structures for Space Applications}

Knitted metal meshes are a key component of Large Deployable Reflectors (LDR) for telecommunication antenna satellites. Both weft and warp knitting methods, are currently used to produced mesh reflector surfaces. In the project Space-R-eflector, a new innovative reflector surface is developed by Large Space Structures GmbH, LSS (concept) and ITA (production technology).

Metal mesh-based reflector surfaces are produced as weft or warp knitted fabrics. Currently, knitting machines with a gauge of E24 or higher are generally used to produce warp knitted metal mesh reflecting surfaces. Tungsten or molybdenum is used as yarn material. [21]. Warp knitted meshes have been previously developed at ITA for use in the surface of large deployable reflector antennae. Currently ITA is investigating warp knitted spacer fabric technology to develop reflector surfaces [22, 23]. This is the first time space fabrics have been considered for use in a reflector surface. The key benefit of the spacer fabric technology is the combination of drapeability (mesh surfaces) and bending stiffness (pile yarn). Thanks to these two initially contradictory capabilities, spacer fabrics are an innovative solution for applications not only in space but in other fields of research as well.

\subsection{Spacer Warp-knitted Non-crimp Fabrics for Construction}

3D spacer fabrics consisting of two textile cover surfaces kept apart by pile yarns are state of the art, e.g. in the field of upholstery. In the z-direction and using predominantly polymer fibers, they retain excellent compression force distribution over thousands of cycles. Their open, sandwich-like structure offers elasticity, insulating properties and has acoustic dampening as well as filtering potential. Technical applications are currently in the field of mattress pads, upholstery, filtration systems and water harvesting methods using so-called fog traps.

For construction applications, spacer warp-knitted non-crimp fabrics are introduced at the Institut für Textiltechnik of RWTH Aachen University (ITA) via a machine development, which can absorb occurring tensile forces in $\mathrm{x}$ - and $\mathrm{y}$-direction, especially in 
concrete structural elements. The 3D-structure consist of two top surfaces made out of pillar $\left(0^{\circ}\right)$ threads, $\left(90^{\circ}\right)$ weft threads, and warp-knitting yarns, which fix the pillar and weft threads together. Pile threads from monofilaments connect both surfaces to each other (Fig. 16). Typical reinforcement materials used are glass, cellulose, aramid, polymer or basalt fibers. Double-needle bar Raschel machines are used for the production of spacer warp-knitted non-crimp fabrics. The warp-knitting elements of the machine are designed symmetrically.

The distance between the two top surfaces can be adjusted by changing the distance between the needle bars. Double Raschel machines consisting of two $90^{\circ}$ weft thread transport systems supply the weft threads to the knitting elements. Special yarn insertion systems allow weft threads to be integrated into the structure in regular or non-regular patterns. Knitting yarns and $0^{\circ}$ yarns are fed to the two knitting units of guide bars. With up to six computer-controlled guide bars currently available, the pattern possibilities offered by this technique are very extensive.

These 3D reinforcement structures have been successfully used as concrete reinforcement in recent years (e.g. from V. Fraas Solutions in Textile GmbH, Helmbrechts, Germany). 3D warp-knitted spacer fabrics offer the advantage that two layers of reinforcement can be integrated into one textile and can be designed variably. Thus, the degree of reinforcement can be freely adjusted via the size of the mesh. The high stiffness of the spacer yarn enables the reinforcement layers to be fixed to each other in a precise position, which is essential for reinforcing concrete. Even a slight deviation of a few millimeters in the reinforcement position leads to unreliable mechanical properties in a concrete component, e.g. a facade panel.

Further applications for 3D spacer fabrics in the construction sector are being researched in the current research project "6d-TEX", funded by the Bundesamt für Bauwesen und Raumordnung, Germany, at the Frankfurt University of Applied Sciences and at the ITA. By combining 3D spacer fabrics with 3D printing, convertible, functional lightweight construction elements are to be realized, which will find their use, for example, as a textile facade shell for shading and insulation.

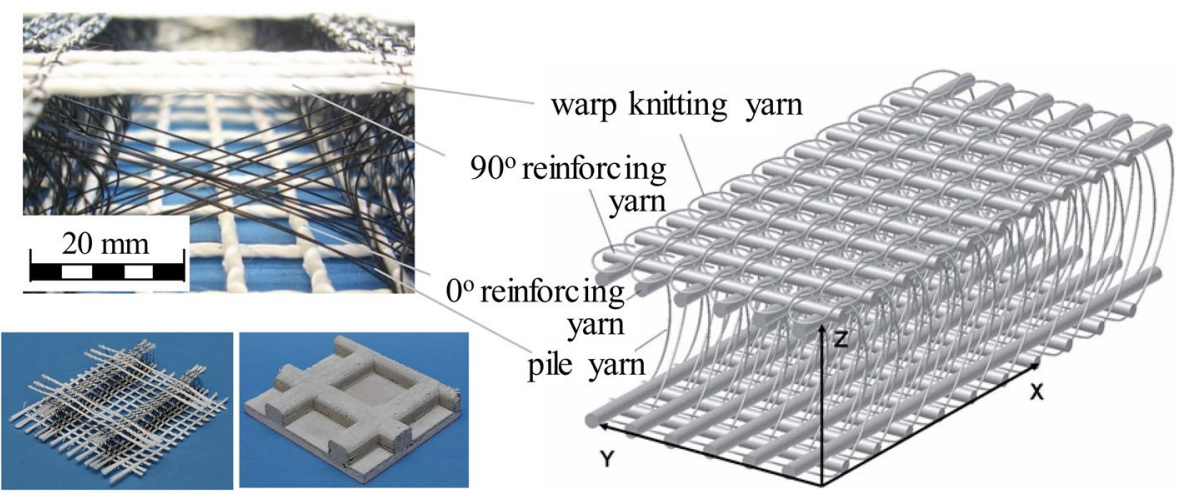

Fig. 16 Spacer warp-knitted non-crimp fabric structure and its use in concrete matrix 


\subsection{FreePreg}

Prepreg components with load-path optimization can currently only be manufactured to a limited extend. Local reinforcements are done by manual layer build-up, resulting in a lot of waste of impregnated semi-finished products. On the other hand, Tailored Fiber Placement (TFP) technology is a key to load-path optimized composite production. TFP is limited to small structures and further limited to dry fiber processing. Using TowPregs has proven impractical due to increased contamination of high-speed TFP embroidery machines.

The FreePreg approach (see Fig. 17) combines the strengths of both prepreg and TFP technologies. The innovation consists in the fact that the novel partially impregnated TFP structures can be used in combination with classic prepregs for the first time. Large-area component sections are built up from prepreg layers at high throughput. TFP reinforcement structures, so-called FreePregs, hence the name, already partially impregnated with epoxy resin, are then applied to the prepreg layers. Consolidation is carried out in the same way as in prepreg processing. This offers advantages over existing process chains. The new step is an impregnation of TFP structures on a roll to roll coating line, fully automated with high throughput.

The FreePreg process therefore separates the impregnation from the embroidery process. By combining large-area prepregs and local TFP reinforcements, large components in particular can be produced with a single-sided mold, resulting in economic advantages over cost-intensive RTM molds. This enables savings in material, handling and manufacturing costs and allows TFP technology to be used in combination with prepreg processing. The implementation in a process chain for the economic production of high-performance fiber composite structures in small to medium series from batch size 1 to 5,000 units/year is targeted. This will enable a reduction in unit costs (up to $€ 8 / \mathrm{kg}$ ) and investment costs (up to $€ 50,000$ per series) compared to established processes. Furthermore, it opens up the possibility for established TFP embroideries to develop further market segments in the CFRP market (up to $8 \%$ of the total CFRP market). It also opens up applicability for TFP in prepreg processing, the most frequently used CFRP manufacturing process (approx. $45 \%$ of the worldwide production volume). Finally, one-sided partial impregnation with solvent-free systems makes it possible for out-of-autoclave-technology and high component quality.

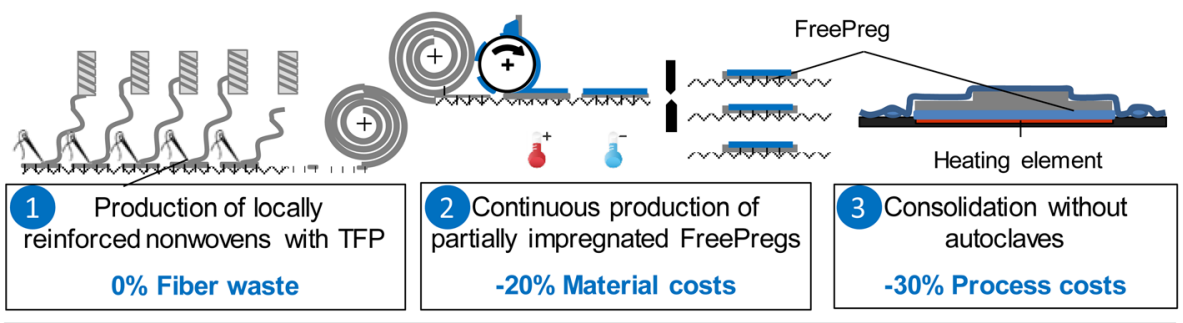

FreePreg enables the combination of the advantages of both processes without high investment costs for SMEs

Fig. 17 Combination of process chains for the production of load-path optimized prepregs 


\subsection{Ceramic Matrix Composite 3D-Braiding}

The integration of fiber reinforcement in ceramics can increase the mechanical properties compared to monolithic ceramics. However, the use of the 2D fiber-reinforced CMC material class remains limited to predominantly thermal applications due to their high shear susceptibility. For example, 2D fiber-reinforced CMCs exhibit a shear strength of $25 \mathrm{MPa}$, which corresponds to only $6 \%$ of the flexural strength (400 $\mathrm{MPa})$ [24]. A significant increase in the mechanical properties of fiber-reinforced ceramics is provided by the 3D-Braiding technology, which was developed at the ITA. Both the machine technology with its components and the process itself have been further modified, so that the handling of brittle ceramic fibers is now possible and allows the production of semi-finished products that are reinforced in all three spatial directions. The 3D-braiding technology adds a reinforcement in the z-direction (see Fig. 18) compared to the 2D-braiding technology, so that interlaminar interfaces inside the braided cross-sections are completely avoided. Consequently, individual layers do not delaminate in the event of damage, as is the case with other reinforcing semi-finished products. This results in an increase of the damage tolerance values for impact stresses and the energies that can be absorbed by the structure (work absorption capacity, vibration damping). Thus, the produced 3D-braided CMC do fulfill the mechanical requirements for structural components in addition to the thermal stress this material can resist. Of particular interest is the use of such 3D-braided components in aircraft turbines. Here, the process efficiency can be increased by means of higher combustion temperatures. Further applications of 3D-braided CMC can be media lines, solar thermal power plants or solid oxide fuel cells.

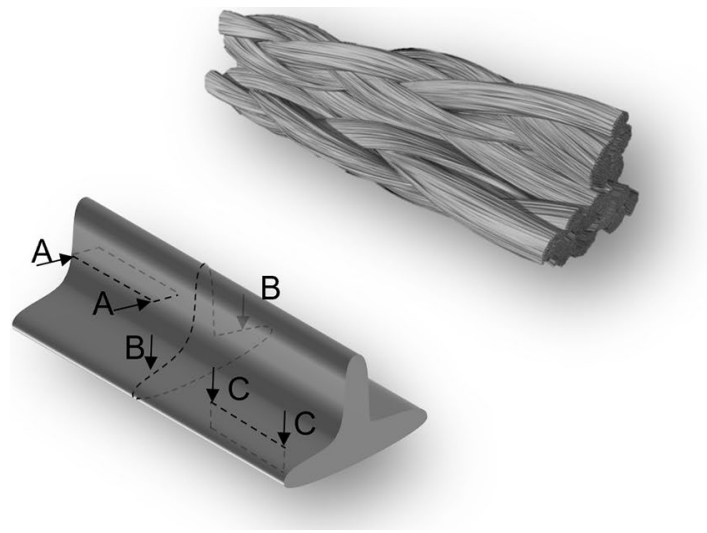

A-A
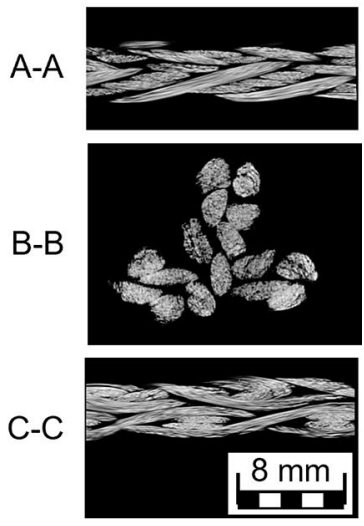

Fig. 18 CT-Scan of a 3D braided T-profile 


\subsection{D Printing On Pre-stressed Textiles}

4D textiles are textiles and textile products that can change their shape or function over time due to the influence of an external stimulus. External stimuli are mainly force or heat [25]. 4D textiles can appear along the whole textile chain, from shape memory fibers, to self-wrinkling weaves and self-folding hybrid materials [26].

At the Institut für Textiltechnik of RWTH Aachen University (ITA) self-folding and self-bending textiles hybrid materials are produced by 3D printing. Mainly fusion deposition modelling (FDM) on prestressed textiles is investigated. Therefore, a filament is extruded on the textile, infiltrates the pores and creates an adhesion-based bond between the materials. The most common filament materials are thermoplastic elastomers such as TPU 95 and thermoplastic PLA. The textile serves as an energy storage medium either through structural elastic properties that can be found in warp and weft knitted fabrics or through material elastic properties to be found in elastane [25].

A shape change from a two-dimensional to a three-dimensional shape appears after releasing the stress from the textile and creates double curved surfaces which represent the lowest possible energy level. More than one equilibrium state is created which allows the hybrid material to shape change between these states after the release of the stress $[25,27]$. The change of shape is activated e.g., by heat which softens the polymer and allows the textile to move.

Applications of 4D textiles in architecture for adaptive shading or acoustic element, as well as interactive car interior are the current focus of research.

\section{Conclusions}

The past and the current efforts at the Institut für Textiltechnik of RWTH Aachen University (ITA) have created innovative products and applications, and new machinery and technologies for 3D fabrics.

Despite the work that was carried out successfully and is still being conducted at present, there are many areas of interest to be explored. Mainly, there is a significant need for digital tools for the design and testing of 3D fabrics. Mechanical properties such as tenacity, static, dynamic and impact behavior and others have to be tested, quantified and provided to composite designers. Furthermore, more fields of applications have to be explored and the machinery has to further developed. Therefore, there is still a great opportunity for interdisciplinary research, discovery and collaboration for the next 30 years to come.

Acknowledgements The authors would like to thank the several funding agencies supporting these various research topics. These include but are not limited to the German Federal Ministry of Economics and Technology (Bundesministerium für Wirtschaft und Technologie), German Federal Ministry of Education and Research (Bundesministerium für Bildung und Forschung) and the European Union.

Funding Open Access funding enabled and organized by Projekt DEAL. The various research topics were funded by several agencies. These include but are not limited to the German Federal Ministry of Economics and Technology (Bundesministerium für Wirtschaft und Technologie), German Federal Ministry of Education and Research (Bundesministerium für Bildung und Forschung) and the European Union.

Data Availability The data, material and code can be found at the following contacts: 


\section{Declarations}

\section{Conflicts of Interest none.}

Open Access This article is licensed under a Creative Commons Attribution 4.0 International License, which permits use, sharing, adaptation, distribution and reproduction in any medium or format, as long as you give appropriate credit to the original author(s) and the source, provide a link to the Creative Commons licence, and indicate if changes were made. The images or other third party material in this article are included in the article's Creative Commons licence, unless indicated otherwise in a credit line to the material. If material is not included in the article's Creative Commons licence and your intended use is not permitted by statutory regulation or exceeds the permitted use, you will need to obtain permission directly from the copyright holder. To view a copy of this licence, visit http://creativecommons.org/licenses/by/4.0/.

\section{References}

1. Roye, A., Stüve, J., Gries, T.: Definition for the differentiation of 2-D and 3-D textiles, Technical Textiles 4/2005 (2005)

2. Hearle, J.W.S.: Definition of 3D textiles in: X. Chen - Advances in 3D Textiles, Cambride: Woodhead Publishing Limited (2015)

3. Perera, Y.S., Muwanwella, R.M.H.W., Fernando, P.R., et al.: Evolution of 3D weaving and 3D woven fabric structures. Fash Text 8, 11 (2021). https://doi.org/10.1186/s40691-020-00240-7

4. Institut für Textiltechnik of RWTH Aachen University

5. Gries, T., Veit, D., Wulfhorst, B.: Textile Fertigungsverfahren - Eine Einführung, 3. aktualisierte und erweiterte Auflage, Hanser (2019)

6. Zhi Hong, S., Shen Hua, Z., Fei Fei, T. et al.: The Study on 3D Weaving Method for Tubular Fabric: Advanced Materials Research. Bd. 331, S.536-540 (2011)

7. Trümper W.: in C. Cherif, Textile Werkstoffe für den Leichtbau, Techniken - Verfahren - Materialien - Eigenschaften, Springer (2011)

8. Schrank, V.: Cost Efficient Production Process of Automotive Interior Textiles using Weft Knitted Spacer Fabrics, Dissertation, RWTH Aachen University (2018)

9. Spencer D.J.: Knitting technology: a comprehensive handbook and practical guide 3. Ed. - Cambridge [u.a]: Woodhead [u.a.] (2001)

10. Liu, Y., Hu, H.: Compression property and air permeability of weft-knitted spacer fabrics. J. Text. Inst. 102(4), 366-372 (2011)

11. Ertekin, G., Marmarali, A.: The compression characteristic of weft knitted spacer fabrics. Tekstil ve Konfesiyon 22(4), 340-345 (2012)

12. Laourine E.: Geflochtene Halbzeuge und Flechttechniken. In: Cherif C. (eds) Textile Werkstoffe für den Leichtbau. Springer, Berlin, Heidelberg (2011)

13. Eichhoff, J., Wendland, B., Gries, T.: Direct three dimensional Tailored Fiber Placement for better mechanical properties of structural parts. Technical Textiles 54, H. 3, S. E146-E147 (2011)

14. Eichhoff, J., Gries, T., Schulz, H.: Verfahren zum Herstellen von dreidimensionalen Faserverbundbauteilen und Halterung hierfür, German Patent DE DE102009041177A1, Date of patent: 24.03.2011 (2011)

15. Fuhrmann, K.: Erhöhung der Flexibilität der Großrundstricktechnologie zur effizienten Fertigung zwei- und dreidimensionaler gestrickter Produkte im Sinne des Mass-Custamization-Ansatzes, Dissertation, RWTH Aachen University (2018)

16. Simonis, K., Gloy, Y.-S., Gries, T.: 3D knitting using large circular knitting machines, 17th World Textile Conference AUTEX 2017: Shaping the Future of Textiles, 29-31 May 2017, Corfu, Greece. IOP Conference Series: Maters. Sci. Eng. (254) (2017)

17. Simonis, K., Gloy, Y.-S., Gries, T.: 3D knitting using large circular knitting machines, In: The Fiber Society (Ed.): The Fiber Society 2017 Spring Conference: Next Generation Fibers for Smart Products, May 17-19, Aachen, Germany (2017)

18. Simonis, K., Gries, T.: 3D formed knitted fabrics by large circular knitting machines, Proceedings of the 8th World Conference on 3D Fabrics and Their Applications, Manchester, UK, 28-29 March (2017)

19. Ashby M.: Materials Selection in Mechanical Design, 4.th edn. Elsevier, Oxford (2011) 
20. Černý, I., Mayer, R.M.: Fatigue of selected GRP composite components and joints with damage evaluation. Composite Structures 94, 664-670 (2012). https://doi.org/10.1016/j.compstruct.2011.08.032

21. Bettermann, I., Raina, A., Gries T.: "A comparison of warp and weft knitting technology for production of metal mesh reflector surfaces" 40th ESA Antenna Workshop - Antenna Developments for Terrestrial and Small-Space Platforms 8-10 October 2019, Noordwijk, The Netherlands (2019)

22. Maghaldadze, N., Nonn, S., Luo, T., Andrade, D., Aggarwal, A., Schoenen, A., Dufour, L., Datashvili, L.: Reduc-ing cost and complexity of small deployable reflectors" 40th ESA Antenna Workshop Antenna Developments for Terrestrial and Small-Space Platforms 8-10 October 2019, Noordwijk, The Netherlands (2019)

23. Bettermann, I., Raina, A., Gries, T., Andrade, D., Nonn, S., Datashvili, L.: Yarn selection for new innovative reflector surface using spacer fabric technology, 6. Nationale Konferenz „Satellitenkommunikation in Deutschland“, 14. Mai 2019 - 15. Mai 2019, Bonn (2019)

24. Rüdinger, A., Pritzkow, W.: Die Entwicklung oxidkeramischer Faserverbundwerkstoffe am Fraunhofer ISC / Zentrum HTL in Zusammenarbeit mit W.E.C. Pritzkow Spezialkeramik Keramische Zeitschrift 65, H. 3, S. 166-169 (2013)

25. Koch, H.C., Schmelzeisen, D., Gries, T.: 4D Textiles Made by Additive Manufacturing on Pre-Stressed Textiles-An Overview. Actuators 10, 31 (2021). https://doi.org/10.3390/act10020031

26. Persson, N.K., Martinez, J.G., Zhong, Y., Maziz, A., Jager, E.W.H.: Actuating Textiles: Next Generation of Smart Textiles. Adv. Mater. Technol. 3, 1-12 (2018). https://doi.org/10.1002/admt.201700397

27. Schmelzeisen, D., Koch, H., Pastore, C., Gries, T.: 4D textiles: Hybrid textile structures that can change structural form with time by 3D printing. Narrow Smart Text. 189-201 (2017). https://doi.org/ 10.1007/978-3-319-69050-6_17

Publisher's Note Springer Nature remains neutral with regard to jurisdictional claims in published maps and institutional affiliations. 